\title{
"Condición humana y ecología integral: Horizontes educativos para una ciudadanía global": Una reseña a un libro que aboga por la unión entre ciencia, filosofía y religión
}

\author{
Maximiliano Reyes Lobos \\ Doctorando en Ética y Democracia \\ Universidad de Valencia, España \\ max.reyeslobos@gmail.com
}

\begin{abstract}
Como citar este artículo: M. REYES-LOBOS ““Condición humana y ecología integral: Horizontes educativos para una ciudadanía global": Una reseña a un libro que aboga por la unión entre ciencia, filosofía y religión" en Palabra y Razón. Revista de Teología, Filosofía y Ciencias de la Religión No16, Diciembre 2019, pp. 99-108 https://doi. org/10.29035/pyr.16.99
\end{abstract}

Tal como es descrito en su prólogo, el libro Condición humana y ecología integral: Horizontes educativos para una ciudadanía global constituye un conjunto de investigaciones llevadas a cabo en el marco del curso denominado "El desafío humanista de la actividad política: Jacques Maritain, el humanismo integral y la UIMP”, celebrado en el verano de 2015 por la Universidad Internacional Menéndez Pelayo. Su autor, el Doctor en Filosofía y catedrático Agustín Domingo Moratalla, lo presenta dividiendo su contenido en siete capítulos, cada uno dirigido al tratamiento de un tema en particular, pero unido a los demás por el hilo conductor de la ecología integral y la condición humana.

El debate y la crítica expuestos en el texto recogen las aportaciones que el conjunto de investigaciones ha realizado a la transformación de la sociedad, entendiéndola como un cambio necesario hacia un escenario en el que la acción humana ha de ser efectivamente una acción y no mero acto de pasividad. Se configura por tanto, un quehacer en el hombre que es integrador de enfoques y que le faculta para aceptar el desafío de construir una relación armónica, tanto con el medio ambiente como consigo mismo.

Un primer aspecto que interesa es la manera en que estas investigaciones son presentadas, constituyéndose como una guía interpretativa y orientadora de la vida moral requerida en tiempos actuales y combinando con simpleza la complejidad 
del conocimiento proveniente de diversas fuentes filosóficas, científicas y eclesiales. Así, el texto repasa los debates relacionados a responsabilidad, educación, ciencias, participación ciudadana en la era de la digitalización, relación entre cristianismo y política, y ofrece una enriquecedora exposición sobre el pensamiento de figuras como Hans-Georg Gadamer, Charles Taylor, Paul Ricoeur, Xavier Zubiri, Adela Cortina, José Antonio Marina, Emmanuel Mounier y el Papa Francisco, sin cuyas contribuciones no se podría comprender a cabalidad la profundidad de la propuesta sobre las transformaciones del ser humano y de la sociedad.

Y es con palabras del primero de estos autores que se plantea la necesidad de contar con un escenario de mayor interdependencia, colaboración y de pertenencia, relevándose la importancia de la relación entre ciencia y filosofía, y criticándose el hecho de que aquella, al dogmatizarse, haya perdido su vínculo con ésta, lo cual repercute en que la responsabilidad sea entendida como una cualidad de la que el saber científico carece. Según esta premisa, la insuficiencia de responsabilidad en la ciencia se traduce en una "incapacidad y falta de necesidad de dar justificación de aquello que ella misma significa en el todo de la existencia humana", idea que como punto de partida, atiende a la relación entre la Ética Hermenéutica y la Teoría de la ciencia, basada en las reflexiones que Gadamer realiza sobre la planificación del futuro. En ellas, "el problema de la planificación del conocimiento en la era de la globalización" (11-12) remite a la confianza que la hermenéutica filosófica mantiene en la ciencia. Claro está, una confianza que no es ciega, sino más bien, razonable y centrada en la responsabilidad sobre la que ésta se asienta y que abarca una crítica tanto al concepto de ciencia en sí mismo, como al uso coloquial de una moral provisional, junto a una propuesta de aplicación de esta última como una categoría mediadora entre racionalización y ética prudencial o sapiencial.

De este modo, cuando se habla del uso crítico que Gadamer realiza al concepto de ciencia, se hace referencia a su reflexión sobre el valor de la filosofía frente al poder absolutista de la ciencia y su campo de aplicación. El principal interrogante apunta a si se debe hablar de filosofía o sobre teoría de la ciencia, o si ha sido un error el plantear la separación entre ciencia y filosofía. Estas preguntas se presentan como un problema de responsabilidad y ponen el acento en que la disociación entre filosofía y ciencia no es un problema propio de una u otra, sino que es una cuestión de aplicación del conocimiento, considerando a la aplicación desde su cariz filosófico.

Gadamer sospecha y desconfía del uso dogmático de la ciencia, sobre todo, el que ha tenido durante la primera mitad del siglo XX. Por ello, ante la necesidad de buscar nuevos horizontes para proveer su uso crítico, nos sitúa en la perspectiva kantiana para hacerlo sin perder la confianza en ella. Remitiéndonos a su obra Verdad 
y método y a la reconstrucción de la noción de verdad, Gadamer nos recuerda que la verdad es entendida como un horizonte de trabajo que moviliza y que permite reconstruir la relación entre filosofía y ciencia desde la interdisciplinaridad y la responsabilidad.

A partir de este punto, el primer capítulo se dedica al significado que para Gadamer tiene la acción de planificar el futuro, situándola como una labor que es propia del Estado y que plantea el interrogante respecto al tipo de moral a trabajar en esta acción y una profundización en la moral provisional. Como resultado, la comunión entre el conocimiento científico y la organización de la vida diaria nos muestra la existencia de tres tipos de moral. A saber, una moral antigua, propia de nuestros padres y abuelos y que por lo tanto, no está necesariamente actualizada; una moral del futuro, relativa a los avances del conocimiento científico y que es catalogada como moral nueva, novísima e innovadora; y una moral provisional, provisoria o proveyente, es decir, que provee. Esta última es la moral que con sus máximas orienta la dirección a seguir en la vida diaria, aun cuando no sean conocidas las particularidades de los avances científicos. La caracterización de estos tres tipos de moral adquiere mayor valía cuando se describen las nuevas tareas que tiene la filosofía moral en un escenario donde el conocimiento científico avanza a pasos agigantados. En esta situación es preciso preguntarse por el modo en el que aplicar estos nuevos y vertiginosos avances de la ciencia, pero haciéndolo sin tener una fe ciega en el proceso; por el contrario, lo que interesa es lograr una aplicación con confianza racional y lograr una planificación que sea mediadora entre la teoría y la praxis.

Para orientar el curso de acción, la propuesta de "una ética planteada desde la naturaleza del diálogo como elemento constitutivo de la condición humana" (24) parece describir una ética no confinada a la mera reflexión sobre las normas que se necesitan en una vida moral, sino que se centra en el tipo de persona que se desea ser. Al respecto, el texto hace hincapié en que la aplicación correcta del conocimiento generado por la ciencia debe contar con un criterio de responsabilidad y estar dirigida hacia la dimensión experiencial de la vida de las personas, pudiéndose garantizar un equilibrio entre lo posible y lo viable. Un equilibrio que aun siendo frágil, se presenta como un punto de encuentro entre ciencia y cultura, entre el conocimiento científico y la cotidianidad, entre la teoría y el significado que la sociedad le otorga.

Ahora bien, dada la aspiración del texto de Agustín Domingo de generar un espacio para el debate y la crítica en torno a la condición humana y la ecología integral, y encontrándose en la relación filosofía-ciencia una clarificadora contextualización, cabe preguntarse por las claves que orientan tal configuración del vínculo entre ser humano y entorno, hallándose en la propuesta presentada por el Papa Francisco en la 
encíclica Laudato si` una primera aproximación. De este marco interpretativo trata el segundo capítulo, en el cual se profundiza en el debate sobre el humanismo en la ética contemporánea y en particular, en las nociones de descuido y de desnaturalización de la técnica.

La invitación de Francisco a repensar el sitial desde el cual nos relacionamos con la naturaleza y con nosotros mismos, se presenta como una propuesta a revisar el paradigma de racionalidad homogeneizador y unidimensional con una crítica al antropocentrismo y la tecnocracia, y como una propuesta de una antropología del cuidado y la responsabilidad. El análisis toma como referencia el enfrentamiento existente entre el poshumanismo y la ecología integral, concibiéndose a la antropología como un problema cuya solución es la resignificación del término cuidado. Esta resignificación se describe como una equivalencia entre cuidado y responsabilidad, en la cual la reflexión filosófica sobre el ser humano y la naturaleza de la antropología redunda en una meditación sobre la continuidad biológica y biográfica del hombre en el mundo, entendiendo por mundo, un escenario global y una casa común.

En este sentido, Laudato si es expuesta como una encíclica que debe ser entendida desde el punto de vista de la antropología del cuidado y la responsabilidad, y prestando atención a la diferencia de trato que en ella se le otorga al concepto de técnica versus el concepto de tecnocracia. En relación a lo primero -y al igual que lo señalado por Gadamer en el capítulo anterior-, la caracterización del cuidado debe hacerse per contrarium, es decir, poniendo el acento en las negligencias y en los descuidos del hombre. Mientras, la relación técnica-tecnocracia se caracteriza por definir a esta última como el paradigma de racionalidad con el que se usa la técnica, de lo cual surgen dos posibles vías de aplicación. Por una parte, utilizar la técnica como una herramienta para ejercer dominio y control sobre el mundo y por otra, como una herramienta que esté al servicio del ser humano y de toda la comunidad biótica. La crítica a la técnica como tecnocracia no se traduce en una crítica a toda la modernidad, sino sólo a ciertas prácticas antropológicas que han demostrado ser negligentes en el cuidado del mundo. Por tanto, no debe ser entendida como una propuesta de su anulación, sino como una reflexión sobre su uso al servicio de la casa común y no a la inversa.

La fundamentación filosófica que ha guiado a Francisco en sus esfuerzos por redefinir la condición humana y su relación con la naturaleza, es descrita en el texto como una profunda reflexión en torno a la necesidad de abandonar la desmesura del extractivismo y dar paso a la recepción y gratitud para con la naturaleza. A partir de ello, Agustín Domingo plantea claves interpretativas que ayudan a comprender el proceso por el cual la encíclica critica el antropocentrismo moderno y posmoderno. 
La expresión del sí mismo en la relación con el mundo desarrollada por Ricoeur; el diagnóstico de la situación de deterioro de la naturaleza entendido como un deterioro del mundo, en tanto que deterioro de la naturaleza y del ser humano como un todo unificado; el uso que el ser humano ha hecho de la técnica, generando "debilitamiento, fragmentación, desvinculación y descuido de los lazos de la familia humana" (44) y las dimensiones de responsabilidad trabajadas por Xavier Zubiri y que apuntan a la responsabilidad retrospectiva que define la raíz humana de la crisis, a la responsabilidad respectiva en la que se sitúa la ecología integral, y a una responsabilidad prospectiva, relativa al cuidado que debemos tener para con las generaciones futuras, son sólo algunos ejemplos.

Luego, reconociendo el aporte que Teilhard de Chardin hace a la encíclica, se afirma que no hay que hacer lecturas reduccionistas y materialistas de la vida biológica, sino que ampliar la perspectiva hacia toda la biodiversidad y la creación en su conjunto. Los esfuerzos por reconstruir una ética del trabajo que lo conciba como una actividad transformadora y propia del ser humano en su intervención en la realidad, sumada a la propuesta de Francisco de separar la política de la economía por medio de un proceso de naturalización, apuntan a lo mismo. En suma, por lo que se vela es por reconocer el valor de la calidad de vida de las personas y situar a la técnica al servicio del ordenamiento real del modelo de desarrollo global.

El capítulo tercero exhibe una aproximación al debate y la crítica en torno a la condición humana y la ecología integral que amplía los horizontes del planteamiento expuesto en el apartado anterior, considerando a la ciudadanía y a la edad secular como protagonistas de la transformación y presentando una interpretación transdisciplinaria que convierte a la encíclica Laudato si en un texto dirigido a todas las personas y no únicamente a quienes pertenecen a la comunidad eclesiástica. De este modo, la encíclica es tratada como un texto fundacional que reconoce que la reconstrucción de la relación con la naturaleza debe hacerse desde la razón y desde la cultura; desde la comunión entre el consenso científico ante la crisis ambiental y la invitación a toda la humanidad a generar un cambio.

En este contexto, no es de extrañar que se denomine a Francisco como un representante del biopersonalismo, postura que en el texto se caracteriza por no desentenderse de la relación del hombre con la naturaleza y que en coherencia, le indica a los bioconservadores no desatender la justicia social y le recuerda a los poshumanistas que el ser humano es un sujeto en y con la naturaleza, por lo cual la tecnología debe estar al servicio de esta relación. Se configura una definición integral que en la línea de lo trabajado por Jacques Maritain, no excluye en su configuración a ninguna dimensión de la vida humana: aquello que es integral se traduce en la acción responsable y el cuidado de las personas, del medio ambiente, de las comunidades, de la casa y de todo lo existente. 
Para clarificar aún más la transición desde un humanismo integral a una ecología integral, Agustín Domingo expone tres hipótesis a modo de explicación. En primer lugar, señala que esta transición surge como respuesta a la necesidad de una propuesta integral que no se identifique con ningún partido político y que vaya más allá de los contextos sociopolíticos iberoamericanos, que es donde se ha habituado el uso del humanismo integral. Como segunda hipótesis, describe cómo los ecologistas y ambientalistas se enfocan en una ecología naturista o medioambientalista, sin utilizar a la ecología integral como concepto ni incorporando la cultura ni la filosofía de la historia en sus postulados y acciones. Al respecto, la conversión hacia una ecología integral unifica los enfoques de cada uno y aporta criterios para lograr justicia social a través del actuar ecológico. En tercera instancia, el paso de un humanismo integral a una ecología integral responde a un debate filosófico entre humanismo y ecologismo, caracterizado por posiciones a favor y en contra. El repaso de estas críticas nos sitúa en los planteamientos de aquellos que como Sartre, defienden el humanismo, y en las ideas de autores como Heidegger, Nietzsche o Foucault, que lo cuestionan.

Más adelante, el capítulo describe tres modelos de ecología presentes tanto en la encíclica como en la reflexión filosófica contemporánea, para luego caracterizar a la ciudadanía ecológica que se configura en torno a la noción de integralidad. Lo relevante en este punto es el cuestionamiento a los modelos de racionalidad y de desarrollo existentes y la invitación que el autor realiza a reflexionar sobre nuevas posibilidades de comunión entre hombre y naturaleza. Posibilidades de las cuales surgiría una ciudadanía ecológica que se configura en torno a la noción de integralidad, con una profunda renovación cultural y valórica sobre la que se construye el futuro y sustentada en una ética del cuidado aplicable en todos los ámbitos del quehacer humano, "desde lo más íntimo y pequeño hasta lo más público y global” (66).

El capítulo cuarto comienza su reflexión recordando el artículo publicado en 1978 por el profesor Augusto Hortal, titulado La democracia como institucionalización de una utopía, en el cual se trata la dimensión utópica de la democracia y el valor de las instituciones como realidades que son simultáneamente estables y cambiantes. Además de su contenido, lo que hace destacar al artículo de Hortal es su aparición casi simultánea con la Constitución Española de 1978, lo que unido al contexto sociopolítico que se da desde aquel entonces y hasta 2015, lo convierte en un hilo conductor para analizar el movimiento $15-\mathrm{M}$ y el paso de la protesta a la propuesta como fenómeno resultante de la combinatoria de las dimensiones utópica e institucional de la democracia.

Sin embargo, este cuarto capítulo va más allá y describe un tercer movimiento que surge con posterioridad al de protesta-propuesta y que se caracteriza por el paso de la propuesta al producto, o lo que es lo mismo, del discurso a la acción. Al respecto, 
la importancia del producto como acción cívica, o más específicamente, como una virtud cívica, radica en que nace de una doble reflexión, siendo la primera de ellas una que combina tres modos complementarios de entender el quehacer cívico. A saber, a) la caracterización del populismo de los indignados -en la que Ernesto Laclau y Chantal Mouffe son nombres clave, b) las propuestas de Hessel y José Luis Sampedro relativas al tiempo de indignación, a la interpelación a los jóvenes y la promoción de una esperanza no violenta, y c) el movimiento de los indignados investigado por la Fundación Encuentro, cuyo informe llamado Una nueva ciudadanía necesaria es identificado por Agustín Domingo como una integración académica del 15-M que recoge los debates que se han dado en dicho campo "sobre el perfeccionamiento de la democracia y cómo se entiende el paso de la protesta a la propuesta" (84).

La segunda parte de la doble reflexión sobre el paso hacia la virtud cívica plantea una crítica al análisis de la indignación por no exhibir una reflexión antropológica sobre "las relaciones humanas, sobre el dinamismo emocional en las teorías de la ciudadanía o sobre el valor de lo nuevo" (92). Frente a ello, las pautas ético-políticas definidas por el autor se presentan como una herramienta para el discernimiento con la cual facilitar el diagnóstico de la indignación experimentada y desarrollarla de un modo saludable. De manera similar, los conceptos trabajados en los capítulos siguientes apuntan a la configuración de una ciudadanía activa, manteniéndose como eje la asunción de responsabilidad en un diálogo entre ciencia y filosofía que ha de ser fluido y con posibilidades de aplicación en escenarios multidisciplinarios. En esta línea, el capítulo quinto se enfoca en el análisis y la reflexión sobre el aprendizaje-servicio (APS) como propuesta educativa.

De acuerdo con lo expuesto por Agustín Domingo, en términos generales, el APS "combina procesos de aprendizaje con servicio a la comunidad en proyectos educativos que promueven una transformación social" (101). Se constituye en una filosofía de educación que orienta a las instituciones educativas a presentarse como organizaciones abiertas a las necesidades sociales. Además, como filosofía que apela a la transformación social, entiende que en este proceso es clave la capacitación a nivel personal y el logro de un desarrollo integral y sostenible. Para analizar las claves éticas del APS, el capítulo expone primeramente una contextualización histórica y luego un marco filosófico, construyéndose un panorama clarificador sobre el positivo impacto que tiene esta propuesta educativa en la sociedad.

El marco histórico consiste en una articulación de valores y ciudadanía, yendo un paso más allá de la separación que se realiza de ambos conceptos cuando se les trata desde la educación en valores y desde la educación para la ciudadanía, respectivamente. Con este punto de vista integrador, el aprendizaje se plantea como un proceso de diálogo en espacios públicos y que moviliza a la totalidad de la persona 
y no únicamente al cerebro o la memoria. La conjugación entre la tradición griega y la tradición académica renacentista se manifiesta en este capítulo como una revisión de los horizontes humanistas que incorpora a su vez, los aportes de las encíclicas de Benedicto XVI y Francisco, quienes en coherencia con lo expuesto con anterioridad en el texto, describen la necesidad de una ciudadanía activa, preocupada y ocupada, y partícipe de los procesos socioeducativos de su tiempo.

Respecto al marco filosófico, la reflexión gira en torno a la generación y transmisión de conocimiento en y junto a la sociedad, con lo cual se muestra la importancia de que las universidades se vinculen con la comunidad y se creen espacios de co-construcción de conocimiento, siendo la responsabilidad y el compromiso actitudes fundamentales en el servicio comunitario. Es de esta forma que el APS se constituye como una "iniciativa que fomenta la transdisciplinariedad y la sostenibilidad como competencias transversales para todos los niveles educativos" (108).

Cabe destacar que, si bien, los aportes filosóficos a este marco pertenecen a pensadores como Jonas, Gadamer, Buber o Weber, el autor explicita que son las ideas de Emmanuel Mounier las que clarifican la importancia del compromiso "como una de las estructuras básicas del universo personal" (110). A partir de ello, el capítulo se ocupa de una propuesta que facilita la comprensión de las ideas centrales del aprendizaje-servicio, planteando conceptos clave y una caracterización de las principales actitudes que a juicio del autor, son a las que se adscribe la comunidad educativa cuando se enfrenta a la propuesta del APS.

A continuación, el capítulo sexto titulado De la neuroeducación a la ecología integral: La oportunidad de una educación civica global, plantea en su introducción la tensión que hay entre patriotismo y cosmopolitismo, partiendo con la referencia al texto de Alasdair MacIntyre ¿Es el patriotismo una virtud?, y describiendo luego cómo los educadores hacen eco de sus reflexiones y se centran en dicha tensión como un enfrentamiento entre lo local y lo global. Frente a esta disputa, Agustín Domingo expone una solución que proviene de las organizaciones cívicas y describe la transformación que ha tenido la ciudadanía en su actuar y en su identidad. Siendo la educación cívica global un elemento transversal en el análisis, el texto busca responder a los cambios y desafíos del nuevo horizonte cultural, prestando atención a dos referencias clave. Por una parte, a los Objetivos para el Desarrollo Sostenible (ODS) que se presentan como una propuesta de solución integral, y por otra, a la Ecología Integral, que se basa en el cuidado del medio ambiente natural y de la "ecología interior de los seres humanos" (123). 
Respecto a la neuroeducación y la neuroética global, el capítulo trata sobre tres aspectos fundamentales a la hora de dar el paso de una a la otra. Nos referimos a los deberes de las neurociencias, a las bases y fundamentos de una libertad condicionada, y al giro en la concepción de la inteligencia, pasando de la inteligencia artificial a la inteligencia maternal. La crítica al cerebrocentrismo y al deslumbramiento por todo lo que use neuro como prefijo se complementa con un reconocimiento de los avances y aportes a la educación que las neurociencias han dado y pueden dar, entendiendo que hay infinidad de oportunidades para la colaboración con las prácticas educativas. $\mathrm{Al}$ respecto, el análisis realizado por el profesor José Antonio Marina es revelador.

Así mismo, el capítulo expone una crítica el reduccionismo que surge al confundirse la pregunta por las bases neurobiológicas con la pregunta por el fundamento. La preponderancia de profundizar en este reduccionismo es debido a que redunda en una concepción de libertad condicionada a la estructura cerebral, desconociendo que la "conciencia humana es compleja y no puede reducirse a su dimensión ejecutiva" (127). Por tanto, la necesidad de una reflexión sobre la relación entre neurociencias y educación se plantea como un punto de encuentro para la configuración de una educación cívica global responsable y que como tal, actúe como mediadora del desarrollo equitativo de las funciones computacionales y ejecutivas de la inteligencia humana.

Dado el trasfondo ético del asunto, la invitación que hace Agustín Domingo es a ir desde la inteligencia artificial hasta la inteligencia maternal, ampliando la reflexión a otros tipos de inteligencia sin circunscribirse únicamente a las neurociencias. Teniendo como referencia la neuroética global e integral trabajada por Adela Cortina, la propuesta del capítulo se centra en la inteligencia maternal como la clave para lograr este fin, con lo cual la inteligencia artificial abandona su sitial como articuladora de la neuroética global y se destaca cómo la maternidad puede hacer a las personas más inteligentes. En suma, sería el paso hacia una inteligencia maternal y por tanto, una inteligencia basada en el cuidado, el mayor aporte a la comprensión de los procesos neurológicos y de nuestro actuar en sociedad.

El reto que plantea la responsabilidad solidaria y la ciudadanía activa pone énfasis en la necesidad que tiene la educación cívica de reconocerse como el resultado del actuar dialógico inherente al ser humano y de convertirse en una condición universal. A la par, la reflexión filosófica en torno al camino que están tomando los avances de la civilización tecnocientífica, basada en las críticas y aportes de Hans Jonas, Paul Ricoeur, Charles Taylor y Xavier Zubiri, implica la concepción de una responsabilidad que cuestione el curso de los avances, pero que al mismo tiempo permita refundar los cimientos de la justicia social y reconocer la identidad moral que nos define como sujetos sociales. En este sentido, el nexo de este apartado 
con el capítulo siguiente y último, se expresa en el interrogante "¿cómo las obras de misericordia pueden generar dinamismo de regeneración política responsable, conciencia cívica de participación solidaria y cohesión social?" (149).

Un primer esbozo de respuesta nace de la descripción del contexto sociocultural de una ciudad pos-secular y poscristiana en circunstancias de incertidumbre política y del rol de la cristiandad en la actividad cívica. En este punto, el autor pone énfasis en el nuevo horizonte abierto que provee la era de la globalización y la digitalización, en la calidad de los vínculos que surgen en ella y en la caracterización de lo que Charles Taylor denomina como la era secular para describir los efectos que la modernización ha generado en las relaciones entre sociedad y religión. Así, y como respuesta a este diagnóstico, el capítulo recoge los aportes de la filosofía contemporánea en un sentido de aplicabilidad a una ética democrática que reconozca el potencial semántico de las creencias y prácticas religiosas, destacándose ciertas líneas de acción que nutren la trama sociopolítica en términos de justicia, misericordia, paz social y reconocimiento.

Como punto final, tanto para el capítulo como para el libro en su conjunto, Agustín Domingo recoge las principales ideas que el Papa Francisco entrega en relación a la misericordia y su poder transformador de la vida personal y social. Con esto se cierra el círculo de la propuesta integradora que busca ampliar los horizontes educativos para una ciudadanía global; horizontes que vistos los aportes, las críticas y los escenarios de aplicación, son propios de la época en la que nos encontramos y que bien puede denominarse -tal como el autor lo hace-como una Edad ecológica de la moral.

Domingo Moratalla, Agustín. Condición humana y ecología integral: Horizontes educativos para una ciudadanía global. PPC: Madrid, 2017. 176 p. 